\title{
Recurrence and Resonance in the Cubic Klein-Gordon Equation
}

\author{
Ferdinand Verhulst ${ }^{1}$
}

Received: 22 August 2018 / Accepted: 11 January 2019 / Published online: 23 January 2019

(C) The Author(s) 2019

\begin{abstract}
In a number of models for coupled oscillators and nonlinear wave equations primary resonances dominate the phase-space phenomena. A new feature is that in a Hamiltonian framework, the interaction of primary and higher order resonances is shown to be important and can be signaled by using recurrence properties. The interaction may involve embedded double resonance. We will demonstrate these phenomena for the cubic Klein-Gordon equation on a square with Dirichlet boundary conditions using normal form techniques. The results are qualitatively and quantitatively very different from the onedimensional spatial case.
\end{abstract}

Keywords 2-dim nonlinear waves · Hamiltonian · Resonance zones · Double resonance

Mathematics Subject Classification $70 \mathrm{H} 07 \cdot 70 \mathrm{H} 12 \cdot 34 \mathrm{E} 10 \cdot 37 \mathrm{~J} 40$

\section{Introduction}

Boundary value problems for nonlinear wave equation produce in a natural way problems with various resonances. We will consider these problems for a typical case, the cubic KleinGordon equation on a square. Galerkin projection and truncation will in this case lead to finite-dimensional Hamiltonian systems.

Consider the two-dimensional cubic Klein-Gordon equation as formulated in [9]:

$$
u_{t t}-u_{x x}-u_{y y}+u=\varepsilon u^{3}, \quad(x, y) \in[0, \pi] \times[0, \pi],
$$

with smooth initial conditions

$$
u(x, y, 0)=\Phi(x, y), \quad u_{t}(x, y, 0)=\Theta(x, y),
$$

and homogenous Dirichlet boundary conditions zero at the sides of the spatial domain. In a sense the subsequent analysis will be a continuation of [9] with attention to higher order

F. Verhulst

f.verhulst@uu.nl

1 Mathematisch Instituut, Utrecht University, PO Box 80.010, 3508TA Utrecht, Netherlands 
resonance and qualitatively new phenomena. In [9] a rectangle is considered instead of a square producing different constant coefficients in Eq. (1). If we would repeat our analysis for a rectangle, the resonances will be different but the analysis runs along the same lines.

After summarizing the approximation theory for nonlinear wave equations we will indicate the resonances in the case of the 2-dimensional cubic Klein-Gordon equation in Sect. 2. The 1:1 resonances are a basic feature of this problem, they are analyzed in various combinations. Recurrence or lack of it will signal the presence or absence of resonance zones that may complicate the dynamics. Detuned resonances will produce embedded double resonance, see Sect. 2.7. Section 3 outlines the asymptotics of detuning.

\subsection{Approximation of Wave Equations}

We will employ two approximation steps for the cubic Klein-Gordon equation: Galerkin truncation of the system and averaging the resulting finite-dimensional system; they produce an asymptotic estimate for the solution of the initial-boundary value problem.

Suppose that the eigenfunctions of the linearized equation $(\varepsilon=0)$ are $\phi_{k l}(x, y), k, l=$ $1,2, \ldots$ The solution of Eq. (1) can be written as:

$$
u(x, y, t)=\sum_{k, l=1}^{\infty} u_{k l}(t) \phi_{k l}(x, y) .
$$

Substituting this Fourier series into Eq. (1) and taking inner products with the eigenfunction expansion (3) produces with corresponding expansion of the initial values an initial value problem for an infinite system of ODEs. The infinite system is equivalent to the original PDE problem. Suppose that Fourier analysis of the initial conditions (smooth functions of $x, y)$ produces a finite series of $N$ terms or $N$ terms with a rest term that can be neglected. A natural Galerkin approximation $u_{N}$ of the solution of Eq. (1) is a truncation of the series (3) with slightly more than $N$ terms. There are many publications using this method but most of them are concerned with formal approximations, see for an example and more references [7]. The analysis of Krol [6] gives the mathematical approximation theory of both the one- and more-dimensional cases. See also the papers of Bambusi [1, 2] and Fečkan [4] for analysis in the same spirit.

The cubic Klein-Gordon equation on a rectangle was studied by Galerkin-averaging in [9]. The resulting system of ODEs obtained for $u_{N}$ produces an approximation $\tilde{u}_{N}$. The proof by Pals [9] uses suitable Sobolev spaces and gives the error estimate in the sup norm. Explicitly:

$$
\left\|u-\tilde{u}_{N}\right\|=o(1) \quad \text { as } \varepsilon \rightarrow 0,
$$

valid on an interval of time $O(1 / \varepsilon)$. In [9] interesting differences with the one-dimensional spatial case are pointed out; many more resonances may arise like detuned and double resonances.

\subsection{Normalizing Hamiltonian Systems}

Consider the $n$ degrees-of-freedom (dof) Hamiltonian $H(p, q)=H_{2}(p, q)+H_{3}(p, q)+\cdots$ with

$$
H_{2}=\frac{1}{2} \omega_{1}\left(p_{1}^{2}+q_{1}^{2}\right)+\frac{1}{2} \omega_{2}\left(p_{2}^{2}+q_{2}^{2}\right)+\cdots+\frac{1}{2} \omega_{n}\left(p_{n}^{2}+q_{n}^{2}\right) .
$$

The frequencies $\omega=\left(\omega_{1}, \omega_{2}, \ldots, \omega_{n}\right)$ are chosen positive; we can approximate them by rational numbers as the rationals are dense in the set of real numbers but its consequence is 
that we have to discuss detuned resonances. The Hamiltonian terms $H_{j}(p, q), j=3,4, \ldots$ are homogeneous polynomials in $p, q$ of degree $j$. We assume that at least two of the frequencies are close to a first or second order resonance, or frequency ratios 1:2, 1:1 or 1:3. We may have detuning effects to allow for small frequency perturbations. For an exhaustive list of first and second order resonances of three dof Hamiltonians see [12], Tables 10.3-10.4.

In many applications a combination of low and higher order resonances takes place. To avoid this one usually concentrates on the low order resonances neglecting the higher order ones. The purpose of this paper is a more complete theory by exploring the cases of combined low and higher order resonance. As we shall see, the tools will be averaging-normal form theory and the use of the Poincaré recurrence theorem to characterize the dynamics in resonance zones.

A powerful theorem on the stability of Hamiltonian systems in the sense of exponentiallylong time invariance of the actions was formulated and proved by Nekhoroshev [8]. This theorem presupposes steepness of the Hamiltonian and so the absence of first or second order resonances in the system. We cannot use the theorem in our case.

In Eq. (1) a small parameter is present but for general $H(p, q)$ it is convenient to scale the coordinates near the stable origin of the system by putting $p, q \rightarrow \varepsilon p, \varepsilon q$ and dividing by $\varepsilon^{2}$. This leads to the Hamiltonian

$$
H(p, q)=H_{2}(p, q)+\varepsilon H_{3}(p, q)+\varepsilon^{2} \ldots
$$

So $\varepsilon^{2}$ is a measure for the energy with respect to stable equilibrium at the origin. Often we introduce action-angle coordinates $I, \phi$ by the transformation:

$$
q_{i}=\sqrt{2 I_{i}} \sin \phi_{i}, \quad p_{i}=\sqrt{2 I_{i}} \cos \phi_{i}, \quad i=1,2,3,
$$

leading with (6) to

$$
H=\sum_{i=1}^{i=n} \omega_{i} I_{i}+\varepsilon H_{3}+\varepsilon^{2} \ldots \quad \text { and } \quad \frac{d I}{d t}=-\frac{\partial H}{\partial \phi}, \quad \frac{d \phi}{d t}=\frac{\partial H}{\partial I} .
$$

We will also use amplitude-phase coordinates $r, \psi$ with transformations $q_{i}, \dot{q}_{i} \rightarrow r_{i}, \psi_{i}$ :

$$
q_{i}=r_{i} \cos \left(\omega_{i} t+\psi_{i}\right), \quad \dot{q}_{i}=-\omega_{i} r_{i} \sin \left(\omega_{i} t+\psi_{i}\right) .
$$

So, the new variables are $r_{i}(t), \psi_{i}(t)$ where we have to exclude a neighborhood of $r_{i}=0$.

We will introduce near-identity transformations producing normal forms; see [12] for theory and background literature. Prominent terms in the normal forms are produced by the resonances induced by the frequencies $\omega$.

A two dof Hamiltonian system in first or second order resonance has an integrable normal form. The treatment of higher order resonance in [11] is quite general for two dof, for more than two dof the complexity of higher order resonance increases enormously. However, in the case of combined lower and higher order resonance we will, as in [16], consider for interactions the so-called resonance zones where the periodic solutions of the (primary) lower resonance are located.

\subsection{Low and Higher Order Resonance}

We consider the theory from [11] and extend it following [13], see also [12]. Consider first a two dof Hamiltonian system with frequencies $k, l \in \mathbb{N}$ near stable equilibrium. If $k+l>4$ 
its Birkhoff-Gustavson normal form is in action-angle coordinates:

$$
\begin{aligned}
H= & k I_{1}+l I_{2}+\varepsilon^{2}\left(A I_{1}^{2}+2 B I_{1} I_{2}+C I_{2}^{2}\right)+\cdots \\
& +\varepsilon^{k+l-2}\left(D_{1}\left(I_{1}, I_{2}\right)+D_{2}\left(I_{1}, I_{2}\right) \cos (\chi+\alpha)\right),
\end{aligned}
$$

where $A, B, C, \alpha$ are constants, the dots stand for Birkhoff normal form terms (dependent on $I_{1}, I_{2}$ only), $\chi=l \phi_{1}-k \phi_{2}$. The angle $\chi$ plays no part in the normal form to $O\left(\varepsilon^{2}\right)$. Considered as an isolated two dof system, it can be shown that the actions of the corresponding modes $I_{1}, I_{2}$ are constant to $O(\varepsilon)$ on the timescale $1 / \varepsilon^{2}$; with some effort the error is reduced to $O\left(\varepsilon^{2}\right)$ on the timescale $1 / \varepsilon^{2}$.

The combination angle $\chi$ may vary locally in a resonance zone as follows: The resonance manifold $N$ embedded in the compact energy manifold $\mathcal{E}$ is defined by $d \chi / d t=0$ or:

$$
N=\left\{I_{1}, I_{2} \in \mathcal{E} \mid(l A-k B) I_{1}+(l B-k C) I_{2}=0\right\} .
$$

If Eq. (10) has no solution, the resonance manifold $N$ does not exist; in this case the combination angle $\chi$ is timelike, we can average over $\chi$. If $N$ exists, small exchanges of energy will take place between the two dof in a resonance zone located in an $O\left(\varepsilon^{(k+l-4) / 2}\right)$ neighborhood of $N$ (this is an improved estimate based on [13]). The resonance zone contains stable and unstable periodic solutions, the exchange of energy takes place on tori in the resonance zone with timescale $1 / \varepsilon^{-(k+l) / 2}$. In [13] it is also proved that for potential problems $\alpha=0$.

Assuming now more than two dof and that the frequency spectrum contains also first and/or second order frequency ratios. The corresponding low order frequency modes will dominate the phase-flow of higher order resonance except in primary resonance zones where the low order actions do not vary; in these zones the low order short-periodic solutions are located.

In the case of many dof our strategy will be to locate the low order resonance zones (small neighborhoods of the resonance manifolds) and find out whether higher order resonance manifolds exist embedded in these zones; they will be called secondary resonance zones. This can be done analytically using normalization. The phenomenon will be called 'embedded double resonance', see [16]. For the general theory of double resonance see $[3,5]$ and more references there.

\subsection{The Recurrence Theorem}

Consider a dynamical system defined on a compact set in $\mathbb{R}^{n}$ with the property that the flow induced by the system is measure-preserving. Poincaré uses the term volume-preserving for the phase-flow induced by a time-independent Hamiltonian system without singularities on a compact domain, see [10], vol. 3, Chap. 26. Using the invariance of the volume of phase-elements under the flow, it is proved that most orbits return an infinite number of times arbitrarily close to their initial position; this is called recurrence. The recurrence time depends on the specific dynamical system considered, the initial condition chosen and the size of the neighborhood to be revisited. Consider for instance an initial point $P_{0}$ and a ball with radius $d>0$ centered around $P_{0}$. The recurrence theorem states that after a finite time $T_{d}$ an orbit starting in this ball will enter the ball again; there are exceptions for certain initial conditions but the exceptional initial conditions have measure zero.

It is easy to obtain an upper limit $L$ for recurrence times, dependent on the Euclidean distance $d(0)=d_{0}$ to the initial condition. Consider time-independent Hamiltonian (6). Assume that $H_{2}(p, q)$ is Morse at $(p, q)=(0,0)$ and that the quadratic part is definite, so the origin is a stable equilibrium of the equations of motion. In [15] it is argued that: 

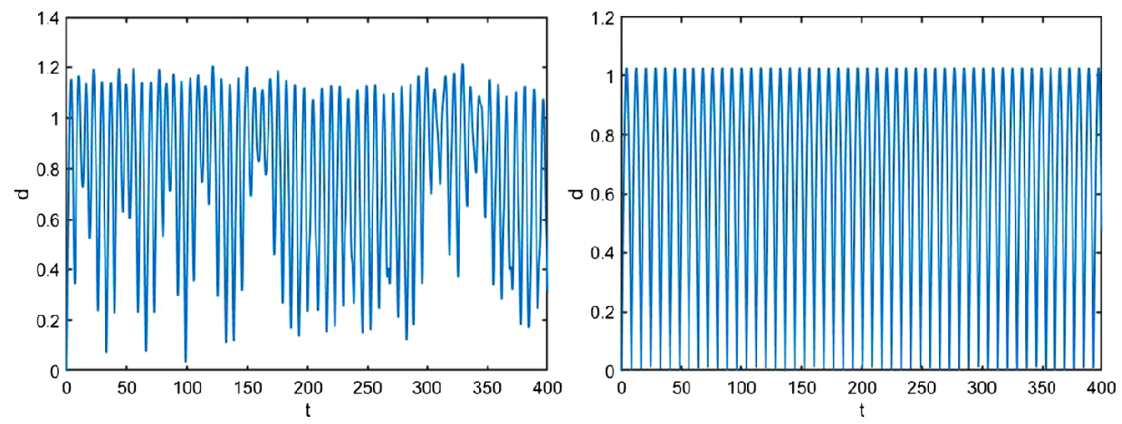

Fig. 1 Recurrence in 2 dof systems for 400 timesteps at energy level $0.1417 \ldots$ (Left) the non-integrable Hénon-Heiles system of Hamiltonian (12) $(a=-1)$ with $x(0)=0.61, \dot{x}(0)=0, y(0)=0, \dot{y}(0)=0.25$ showing irregular behavior of the Euclidean distance $d$ to the initial values. (Right) the integrable case of Hamiltonian (12) $(a=+1)$ with the same initial conditions. The recurrence looks fairly regular

Proposition 1.1 Each orbit near stable equilibrium of the system induced by Hamiltonian (6), except a number of orbits in a set of measure zero, reaches a size $d_{0}$ neighborhood of its initial point with upper bound $L$ of the recurrence time $T_{d_{0}}$ :

$$
L=O\left(\frac{1}{d_{0}^{2 n-1}}\right) \quad \text { as } d_{0} \rightarrow 0 .
$$

To illustrate recurrence we present in Fig. 1 the Euclidean distance to an initial state for the Hénon-Heiles system that has been shown to be non-integrable and an integrable system, both for 2 dof; see for a detailed analysis [14]. The Hamiltonian is:

$$
H=\frac{1}{2}\left(x^{2}+\dot{x}^{2}\right)+\frac{1}{2}\left(y^{2}+\dot{y}^{2}\right)-\frac{1}{3} x^{3}-a x y^{2} .
$$

Doubling the integration time for the Hénon-Heiles system, $a=-1$, produces a similar picture. In the integrable case $a=1$ we have periodic solutions and tori foliating the energy manifold; in this case the recurrence is called regular. We might also call recurrence regular if we have a non-integrable system with complex regions that are relatively small (a precise definition of "regular" is difficult as there are so many different cases).

In the sequel we have often $n=3, d_{0}=0.1$ producing $L=10^{5}$, if $n=4, d_{0}=0.1, L=10^{7}$. The actual Poincaré recurrence times are lower than $L$ but passage of resonance zones can delay recurrence as the orbits will wind around the tori embedded in the resonance zones.

A preliminary test for embedded double resonance can be carried out using the recurrence theorem. Constructing numerical solutions of orbits passing the resonance zones, we expect fairly regular recurrent behavior if these zones contain no or very small resonance manifolds. Complicated and long time recurrent behavior points at motion around tori and other invariant manifolds during passage. In [16] the 1:1:4 resonance and the Fermi-Pasta-Ulam $\alpha$-chain were discussed as examples. Recurrence will be tested by computing the Euclidean distance $d(t)$ to the initial conditions as a function of time.

\section{The Cubic Klein-Gordon Equation}

In the sequel we will consider problems derived from the cubic Klein-Gordon equation (1). The eigenfunctions of the linearized equation on a square are $\phi_{k l}(x, y)=\sin k x \sin l y, k, l=$ $1,2, \ldots$ 


\subsection{Asymptotic Approximations}

The eigenfunction expansion (3) that satisfies the boundary conditions is:

$$
u(x, y, t)=\sum_{k, l}^{\infty} u_{k l}(t) \sin k x \sin l y .
$$

The eigenfunctions $\sin k x \sin l y$ of the linearized Eq. (1) correspond with the eigenvalues

$$
\omega_{k l}^{2}=k^{2}+l^{2}+1 \text {. }
$$

Fourier expansion turns the partial differential equation into an equivalent system of an infinite number of coupled ordinary differential equations of the form

$$
\ddot{u}_{k l}+\omega_{k l}^{2} u_{k l}=\varepsilon f_{k l}(u), \quad k, l=1,2, \ldots
$$

with $f_{k l}(u)$ cubic in $u_{k l}, k, l=1,2, \ldots$. As announced in the Introduction we will employ two approximation steps: Galerkin truncation of the system and averaging the resulting finite-dimensional system. The asymptotic approximation will have a validity on an interval of time of $O\left(\omega_{k l} / \varepsilon\right)$; as we shall see, in practice this estimate is often too pessimistic.

Part of our interest will be on the interaction of the resonant and non-resonant part of the spectrum. The analysis in [9] is quite general, here we illustrate the resonances for the 69 modes with $\omega_{k l}^{2} \leq 100$. Note that all cases with $k \neq l$ produce a 1:1 resonance; the 1:1 resonances turn out to be basic for this problem. We find in addition:

- $1: 3: 3$ resonances with

$$
\begin{aligned}
& k l=11,15,51 ; \omega_{11}^{2}=3, \omega_{15}^{2}=27 \\
& k l=22,48,84 ; \omega_{22}^{2}=9, \omega_{48}^{2}=81 \\
& k l=13,31,77 ; \omega_{13}^{2}=11, \omega_{77}^{2}=99
\end{aligned}
$$

- $1: 1: 3: 3$ resonance

$$
k l=12,21,27,72 ; \omega_{12}^{2}=6, \omega_{27}^{2}=54
$$

- $1: 1: 1$ resonance

$$
k l=55,17,71 ; \omega^{2}=51
$$

- $1: 1: 1: 1$ resonances

$$
\begin{aligned}
& k l=47,74,18,81 ; \omega^{2}=66 \\
& k l=67,76,29,92 ; \omega^{2}=86
\end{aligned}
$$

- Detuned 1:2:2 resonance

$$
k l=33,57,75 ; \omega_{33}^{2}=19, \omega_{57}^{2}=\omega_{75}^{2}=75
$$

$k l=14,41,66 ; \omega_{14}^{2}=\omega_{41}^{2}=18, \omega_{66}^{2}=73$

- Detuned 1:1:2:2 resonance

$$
k l=24,42,19,91 ; \omega_{24}^{2}=21, \omega_{19}^{2}=83
$$

- Detuned 1:1:3:3 resonance

$$
k l=13,31,49,94 ; \omega_{13}^{2}=11, \omega_{49}^{2}=98
$$

- A higher order 3:3:7:7 resonance with $k l=14,49$

The 3:7 resonance turns out to have no resonance manifold in a Galerkin projection as the corresponding combination angle is timelike, so we leave out this case. Note also that the larger $k+l$ is, the smaller the resonance zones become and the larger the interaction timescales are, see Sect. 1.3.

We will start with a sketch of the basic 1:1 resonances; after this we study the dynamics and weak interactions with other resonances. Our focus will be on the interesting case where recurrence highlights more complicated dynamics involving higher order resonance. 


\subsection{The Basic 1:1 Resonances}

Restricting to two modes $u_{k l}, u_{l k}, k \neq l$ we find an infinite number of 1:1 resonances. Galerkin projection produces with $\omega_{k l}^{2}=\omega_{l k}^{2}=\omega^{2}$ the system:

$$
\left\{\begin{array}{l}
\ddot{u}_{k l}+\omega^{2} u_{k l}=\varepsilon \frac{3}{4} u_{k l}\left(\frac{3}{4} u_{k l}^{2}+u_{l k}^{2}\right) \\
\ddot{u}_{l k}+\omega^{2} u_{l k}=\varepsilon \frac{3}{4} u_{l k}\left(\frac{3}{4} u_{l k}^{2}+u_{k l}^{2}\right)
\end{array}\right.
$$

As the size of the parameter $\omega$ is still free it is natural to rescale time $t \rightarrow \tau=\omega t$ and call the rescaled time again $t$. The system becomes:

$$
\left\{\begin{array}{l}
\ddot{u}_{k l}+u_{k l}=\frac{\varepsilon}{\omega^{2}} \frac{3}{4} u_{k l}\left(\frac{3}{4} u_{k l}^{2}+u_{l k}^{2}\right), \\
\ddot{u}_{l k}+u_{l k}=\frac{\varepsilon}{\omega^{2}} \frac{3}{4} u_{l k}\left(\frac{3}{4} u_{l k}^{2}+u_{k l}^{2}\right) .
\end{array}\right.
$$

The dynamics of this system was analyzed in [14] and [9]; it is characterized by an integrable normal form, two unstable normal modes and two resonance zones with periodic solutions. We add some new elements. We exclude the normal modes in the next approximation procedure as we use polar coordinates. Putting, $u_{k l}=r_{1} \cos \left(t+\psi_{1}\right), u_{l k}=r_{2} \cos \left(t+\psi_{2}\right)$, the equations from first order averaging-normalization become (see Sect. 3):

$$
\begin{cases}\dot{r}_{1}=-\frac{\varepsilon}{\omega^{2}} \frac{3}{32} r_{1} r_{2}^{2} \sin 2 \chi_{1}, & \dot{\psi}_{1}=-\frac{\varepsilon}{\omega^{2}} \frac{3}{16}\left(\frac{9}{8} r_{1}^{2}+r_{2}^{2}+\frac{1}{2} r_{2}^{2} \cos 2 \chi_{1}\right), \\ \dot{r}_{2}=+\frac{\varepsilon}{\omega^{2}} \frac{3}{32} r_{1}^{2} r_{2} \sin 2 \chi_{1}, & \dot{\psi}_{2}=-\frac{\varepsilon}{\omega^{2}} \frac{3}{16}\left(\frac{9}{8} r_{2}^{2}+r_{1}^{2}+\frac{1}{2} r_{1}^{2} \cos 2 \chi_{1}\right),\end{cases}
$$

with $\chi_{1}=\psi_{1}-\psi_{2}$. We find:

$$
\frac{d \chi_{1}}{d t}=-\frac{\varepsilon}{\omega^{2}} \frac{3}{32}\left(r_{1}^{2}-r_{2}^{2}\right)\left(\frac{1}{4}-\cos 2 \chi_{1}\right) .
$$

An integral of the normal form (17) is

$$
r_{1}^{2}+r_{2}^{2}=2 E_{1}
$$

with constant $E_{1} \geq 0$; so the normal form is integrable. Note (again) that increasing $k, l$ and so $\omega=\omega_{k l}$, we increase the timescale of validity that characterizes the dynamics. Phaselocked periodic solutions are found in the resonance manifolds and are determined by:

$$
r_{1}=r_{2}=E_{1}, \quad \chi_{1}=0, \frac{\pi}{2}, \pi, 3 \frac{\pi}{2} .
$$

Because of the symmetry of the equations we can obtain exact solutions. The periodic solutions correspond with the solutions $u_{k l}(t)=u_{l k}(t), u_{k l}(t)=-u_{l k}(t)$. They satisfy the equation:

$$
\ddot{u}_{k l}+u_{k l}=\frac{\varepsilon}{\omega^{2}} \frac{21}{16} u_{k l}^{3} .
$$

Substitution in (1) produces a Galerkin two-mode projection of periodic solutions.

An additional feature of the Galerkin projection for the cubic Klein-Gordon equation is that there exist other phase-locked solutions if $\cos 2 \chi_{1}=1 / 4$. Substituting this value into 
system (17) we find heteroclinic invariant manifolds connecting the unstable normal modes. Both the periodic solutions in the resonance zones and the heteroclinic solutions will be a returning feature in what follows.

We can introduce the variable angular momentum $J(t)$ for the $u_{k l}, u_{l k}$ interaction:

$$
J=\dot{u}_{k l} u_{l k}-u_{k l} \dot{u}_{l k}
$$

For $J$ we find from system (16) the equation

$$
\frac{d J}{d t}=-\frac{\varepsilon}{32 \omega^{2}} u_{k l} u_{l k}\left(u_{k l}^{2}-u_{l k}^{2}\right)
$$

We conclude that in the resonance manifolds, where $u_{k l}^{2}=u_{l k}^{2}$, the angular momentum of the $u_{k l}, u_{l k}$ interaction is conserved. The resonance zones correspond with critical points of the angular momentum equation (22).

\subsection{Combining Independent 1:1 Resonances}

Suppose we have a Galerkin truncation with a finite number $M$ of basic 1:1 resonances $k_{i}, l_{i}, k_{i} \neq l_{i}$ for certain indices $k_{i}, l_{i}$. We exclude the special resonance cases as presented in Sect. 2.1: (1:1:1:1), (1:1:2:2) and (1:1:3:3).

With this assumption the $M$ basic 1:1 resonances will be independent of each other. The resulting approximation will be a superposition of the individual basic resonances.

Note that for values of $\omega$ such that $1 / \omega^{2} \leq \varepsilon$ the contribution of such a $1: 1$ resonance will be of order $\varepsilon^{2}$.

\subsection{The First Three Modes}

Apart from the basic 1:1 resonances it is natural to consider the first three modes. The eigenvalues are $\omega_{11}^{2}=3, \omega_{12}^{2}=\omega_{21}^{2}=6$. With the corresponding $u_{11}(t), u_{12}(t), u_{21}(t)$ we propose the three-terms Galerkin truncation:

$$
u_{11}(t) \sin x \sin y+u_{12}(t) \sin x \sin 2 y+u_{21}(t) \sin 2 x \sin y .
$$

Substituting this truncation into Eq. (1) and taking inner products with the eigenfunctions we find the $1: 1$ resonance imbedded in a three dof system. Replacing $\sqrt{6} t$ by $t$ we find:

$$
\left\{\begin{array}{l}
\ddot{u}_{11}+\frac{1}{2} u_{11}=\varepsilon \frac{3}{16}\left(\frac{1}{2} u_{11}^{3}+u_{11} u_{12}^{2}+u_{11} u_{21}^{2}\right), \\
\ddot{u}_{12}+u_{12}=\varepsilon \frac{1}{8}\left(\frac{3}{4} u_{12}^{3}+\frac{3}{2} u_{11}^{2} u_{12}+u_{12} u_{21}^{2}\right), \\
\ddot{u}_{21}+u_{21}=\varepsilon \frac{1}{8}\left(\frac{3}{4} u_{21}^{3}+\frac{3}{2} u_{11}^{2} u_{21}+u_{12}^{2} u_{21}\right) .
\end{array}\right.
$$

The three normal modes are solutions of system (24). Putting $u_{11}(t)=0$, the system has a two dof (4-dimensional) invariant manifold, the dynamics of which was analyzed before (Sect. 2.2). It is simple to repeat part of the calculation to assess the role of $u_{11}$; the frequency ratios are $\frac{1}{2} \sqrt{2}: 1: 1$ so the first mode is not close to resonance with the other two modes. Excluding the normal modes, averaging-normalization for amplitudes and phases $r, \psi$ from 

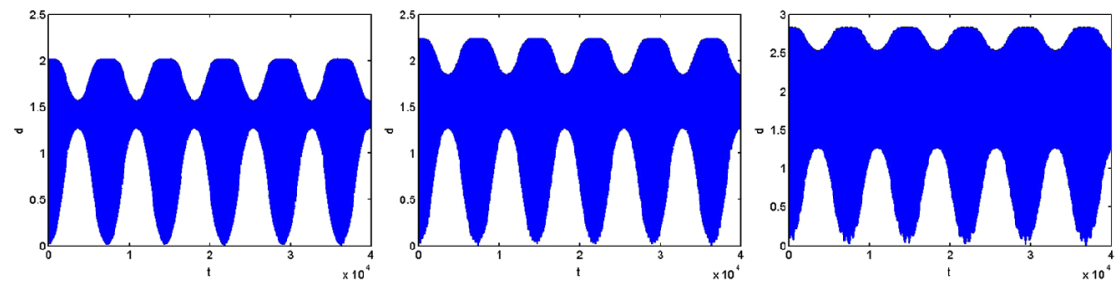

Fig. 2 Recurrence in the two-dimensional Klein-Gordon equation, $\varepsilon=0.1$, for 40000 timesteps. The Euclidean distance $d$ to the initial values $u_{12}(0)=0.1, u_{21}(0)=1$, initial velocities zero, for system (24) is shown in the case of passage through the resonance zones. Left the case $u_{11}(0)=0.1$ that is close to pure 1:1 resonance, middle $u_{11}(0)=0.5$ and right $u_{11}(0)=1$. The dark areas (on a screen blue) describe large oscillations resulting in large oscillations of $d$. The action $I_{1}$ (not shown) shows very small variations $(0.01)$, the recurrence is fairly regular and hardly depends on $u_{11}(0)$

(8) produces with

$$
\begin{aligned}
& u_{11}=r_{1} \cos \left(\frac{1}{2} \sqrt{2} t+\psi_{1}\right), \quad u_{12}=r_{2} \cos \left(t+\psi_{2}\right), \quad u_{21}=r_{3} \cos \left(t+\psi_{3}\right), \\
& \chi_{1}=\psi_{2}-\psi_{3}: \\
& \begin{cases}\dot{r}_{1}=0, \quad \dot{\psi}_{1}=-\frac{\varepsilon}{64} 3 \sqrt{2}\left(\frac{3}{4} r_{1}^{2}+r_{2}^{2}+r_{3}^{2}\right), \\
\dot{r}_{2}=-\frac{\varepsilon}{64} r_{2} r_{3}^{2} \sin 2 \chi_{1}, \quad \dot{\psi}_{2}=-\frac{\varepsilon}{64}\left(\frac{9}{4} r_{2}^{2}+3 r_{1}^{2}+r_{3}^{2}\left(2+\cos 2 \chi_{1}\right)\right), \\
\dot{r}_{3}=+\frac{\varepsilon}{64} r_{2}^{2} r_{3} \sin 2 \chi_{1}, \quad \dot{\psi}_{3}=-\frac{\varepsilon}{64}\left(\frac{9}{4} r_{3}^{2}+3 r_{1}^{2}+r_{2}^{2}\left(2+\cos 2 \chi_{1}\right)\right) .\end{cases}
\end{aligned}
$$

The solutions of system $(25)$ are $O(\varepsilon)$ approximations of the amplitudes and phases from system (24) valid on the timescale $1 / \varepsilon$; note that $\varepsilon$ plays here the part of $\varepsilon^{2}$ in expansion (6). It was proved in [9] that using these approximations in the Galerkin expansion (13) with corresponding initial conditions produces an asymptotic approximation as $\varepsilon \rightarrow 0$ of Eq. (1) on the same timescale $1 / \varepsilon$.

System (25) has the integrals $r_{2}^{2}+r_{3}^{2}=2 E_{1}$ and $r_{1}(t)=r_{1}(0)$. For the combination angle $\chi_{1}$ we have:

$$
\frac{d \chi_{1}}{d t}=-\frac{\varepsilon}{64}\left(r_{2}^{2}-r_{3}^{2}\right)\left(\frac{1}{4}-\cos 2 \chi_{1}\right) .
$$

Apart from the normal modes of system (24), four families of iso-energetic quasi-periodic solutions parametrized by $r_{1}(0)$ arise of system (25) if $\sin 2 \chi_{1}=0, r_{2}=r_{3}$. The two periods of each family depend on the initial conditions i.e. the energy level. They correspond with approximate quasi-periodic standing waves of Eq. (1).

As in the case of Sect. 2.2 there exist other phase-locked solutions if $\cos 2 \chi_{1}=1 / 4$. Substituting this value into system (25) we find heteroclinic invariant manifolds connecting the $u_{2}, u_{3}$ normal modes. For instance for $r_{2}$ from the equation

$$
\dot{r}_{2}=-\frac{\varepsilon}{64} c r_{2}\left(2 E_{1}-r_{2}^{2}\right)
$$

with $\cos 2 \chi_{1}=1 / 4, c=\sin 2 \chi_{1}$.

The resonance zones $M$ on the energy manifold are neighborhoods of the quasi-periodic solutions given by $\sin 2 \chi_{1}=0, r_{2}=r_{3}$ corresponding with $\sin 2 \chi_{1}=0, u_{12}=u_{21}$. Consider as an example the zone $r_{2}^{2}=r_{3}^{2}=E_{1}, \chi_{1}=0$ and $r_{1}(0)$ to be chosen. Passage of the resonance zone is shown in Fig. $2 ; I_{1}(t)$ shows variations of order 0.01 . The dynamics in the 
resonance zones shows regular quasi-periodic behavior near the periodic solutions as follows from our analysis, there is hardly any exchange of energy with mode $u_{11}$; the numerics shows that details of recurrence are slightly affected by the choice of $u_{11}(0)$. Choosing as recurrence criterion $d_{0}=0.1$, the recurrence times are nearly 8000 timesteps, the interval of time to let $d$ approach zero again and again.

The equations for the phases in $M$ are:

$$
\dot{\psi}_{1}=-\frac{\varepsilon}{64} 3 \sqrt{2}\left(\frac{3}{4} r_{1}(0)^{2}+2 E_{1}\right), \quad \dot{\psi}_{2}=\dot{\psi}_{3}=-\frac{\varepsilon}{64}\left(3 r_{1}(0)^{2}+\frac{21}{4} E_{1}\right) .
$$

It turns out that the variations of the three actions when starting in $M$ are of size 0.01 on an interval of 40000 timesteps.

As the frequency ratio $\omega_{11}: \omega_{12}$ is close to 7:10, one could look for the 7:10 higher order resonance in the resonance zones. As we find from Eq. (27) that the combination angle $\left(10 \psi_{1}-7 \psi_{2}\right)$ is timelike, this higher order resonance does not arise.

More insight can be obtained by returning to the Galerkin equations (24). The symmetry of the equations suggest that $u_{12}^{2}(t)=u_{21}^{2}(t)$ satisfies system (24). The equation for $u_{12}$ is in this special case:

$$
\ddot{u}_{12}+u_{12}=\frac{\varepsilon}{16} u_{12}\left(\frac{7}{2} u_{12}^{2}+3 u_{11}^{2}\right) .
$$

We can introduce the variable angular momentum $J(t)$ for the $u_{12}, u_{21}$ interaction by (21). For $d J / d t$ we find from system (24) again Eq. (22). It is remarkable that the equation for $J$ does not depend on $u_{11}$, we draw conclusions similar to those in Sect. 2.2. In the resonance zones, where $u_{12}^{2}=u_{21}^{2}$, angular momentum of the $u_{12}, u_{21}$ interaction is conserved. The resonance zones correspond with critical points of the angular momentum equation (22). In addition we can derive from system (24) the equation for $u_{11}(t)$ in $M$ :

$$
\ddot{u}_{11}+\frac{1}{2} u_{11}=\frac{\varepsilon}{32} u_{11}\left(3 u_{11}^{2}+12 u_{12}^{2}\right) \text {. }
$$

In $M$ we expect $u_{11}(t)$ to vary very little.

\subsection{Coupled 1:1 Resonances with Different Frequencies}

Considering a truncation to 4 modes generated by $k_{1} l_{1}, k_{2} l_{2}\left(k_{1} \neq l_{1}, k_{2} \neq l_{2}\right)$ we may find interaction if a resonance exists between the frequencies $\omega_{k_{1} l_{1}}, \omega_{k_{2} l_{2}}$. As a typical example we consider the case of the 1:1:3:3 resonance with $k l=12-21-27-72 ; \omega_{12}^{2}=6, \omega_{27}^{2}=$ 54. Coupled 1:1 resonances were discussed in [9] without the additional 1:3 resonance.

Substituting the 4-mode truncation into Eq. (1), taking inner products with the eigenfunctions and replacing $\sqrt{6} t$ by $t$ we find the four dof system:

$$
\left\{\begin{array}{l}
\ddot{u}_{12}+u_{12}=\varepsilon \frac{1}{8} u_{12}\left(\frac{3}{4} u_{12}^{2}+u_{21}^{2}+u_{27}^{2}+u_{72}^{2}\right), \\
\ddot{u}_{21}+u_{21}=\varepsilon \frac{1}{8} u_{21}\left(\frac{3}{4} u_{21}^{2}+u_{12}^{2}+u_{27}^{2}+u_{72}^{2}\right), \\
\ddot{u}_{27}+9 u_{27}=\varepsilon \frac{1}{8} u_{27}\left(u_{12}^{2}+u_{21}^{2}+\frac{3}{4} u_{27}^{2}+u_{72}^{2}\right) . \\
\ddot{u}_{72}+9 u_{72}=\varepsilon \frac{1}{8} u_{72}\left(u_{12}^{2}+u_{21}^{2}+\frac{3}{4} u_{72}^{2}+u_{27}^{2}\right) .
\end{array}\right.
$$

The four normal modes are solutions of system (29). Other exact solutions carry over from Sect. 2.2:

$$
u_{12}^{2}=u_{21}^{2}, \quad u_{27}^{2}=u_{72}^{2},
$$


and again we can introduce angular momentum; we will discuss the differences with the case of one 1:1 resonance. Averaging-normalization of system (29) will show that the interaction between the two 1:1 resonances takes place between the angles. Introducing polar coordinates (excluding the normal modes) we associate $u_{12}$ with $r_{1}, \psi_{1}$ etc. and find to first order:

$$
\begin{cases}\dot{r}_{1}=-\varepsilon \frac{1}{64} r_{1} r_{2}^{2} \sin 2 \chi_{1}, & \dot{\psi}_{1}=-\varepsilon \frac{1}{32}\left(\frac{9}{8} r_{1}^{2}+r_{2}^{2}+\frac{1}{2} r_{2}^{2} \cos 2 \chi_{1}+r_{3}^{2}+r_{4}^{2}\right), \\ \dot{r}_{2}=+\varepsilon \frac{1}{64} r_{1}^{2} r_{2} \sin 2 \chi_{1}, & \dot{\psi}_{2}=-\varepsilon \frac{1}{32}\left(\frac{9}{8} r_{2}^{2}+r_{1}^{2}+\frac{1}{2} r_{1}^{2} \cos 2 \chi_{1}+r_{3}^{2}+r_{4}^{2}\right), \\ \dot{r}_{3}=-\varepsilon \frac{1}{192} r_{3} r_{4}^{2} \sin 2 \chi_{2}, & \dot{\psi}_{3}=-\varepsilon \frac{1}{96}\left(r_{1}^{2}+r_{2}^{2}+\frac{9}{8} r_{3}^{2}+r_{4}^{2}+\frac{1}{2} r_{4}^{2} \cos 2 \chi_{2}\right), \\ \dot{r}_{4}=+\varepsilon \frac{1}{192} r_{3}^{2} r_{4} \sin 2 \chi_{2}, & \dot{\psi}_{4}=-\varepsilon \frac{1}{96}\left(r_{1}^{2}+r_{2}^{2}+r_{3}^{2}+\frac{1}{2} r_{3}^{2} \cos 2 \chi_{2}+\frac{9}{8} r_{4}^{2}\right),\end{cases}
$$

with $\chi_{1}=\psi_{1}-\psi_{2}, \chi_{2}=\psi_{3}-\psi_{4}$. We find the integrals:

$$
r_{1}^{2}+r_{2}^{2}=2 E_{1}, \quad r_{3}^{2}+r_{4}^{2}=2 E_{2} .
$$

For the combination angles we find:

$$
\frac{d \chi_{1}}{d t}=-\varepsilon \frac{1}{64}\left(r_{1}^{2}-r_{2}^{2}\right)\left(\frac{1}{4}-\cos 2 \chi_{1}\right), \quad \frac{d \chi_{2}}{d t}=-\varepsilon \frac{1}{192}\left(r_{3}^{2}-r_{4}^{2}\right)\left(\frac{1}{4}-\cos 2 \chi_{2}\right)
$$

Eliminating $r_{2}$ from the equation for $\chi_{1}, r_{1}$ from the equation for $\chi_{2}$, we obtain equations for $d r_{1} / d \chi_{1}$ and $d r_{2} / d \chi_{2}$ that can be integrated producing 2 extra integrals; we leave out the cumbersome expressions. We conclude that the normal form system (30) is integrable.

If $r_{3}(0)=r_{4}(0)=0$ we find 4 periodic solutions in a submanifold from the conditions $r_{1}^{2}(0)=r_{2}^{2}(0)=E_{1}, \sin 2 \chi_{1}=0$. If $r_{3}(0) r_{4}(0) \neq 0$, the approximate solutions in the resonance zone $r_{1}^{2}(0)=r_{2}^{2}(0)=E_{1}, \sin 2 \chi_{1}=0$ are in general not periodic anymore as $u_{12}, u_{21}$ are periodic with period depending on $E_{1}$ and $E_{2}$, but $u_{27}, u_{72}$ are in general not periodic. An analogous reasoning applies when considering $r_{1}(0)=r_{2}(0)=0$ and the resonance zone $r_{3}^{2}(0)=r_{4}^{2}(0)=E_{2}, \sin 2 \chi_{2}=0$. A different case arises when the resonance zones intersect, we have a double resonance. In this case we find quasi-periodic solutions with two periods depending on $E_{1}, E_{2}$. We can characterize the intersection by putting $u_{12}^{2}=u_{21}^{2}, u_{27}^{2}=u_{72}^{2}$ in system (29) and applying averaging-normalization to the resulting equations. Although there is angle-coupling between the modes, the 1:3 resonance is not present in the intersection.

\subsection{Coupled 1:1 Resonances, the 1:1:1:1 Resonance}

Consider now a truncation to 4 modes generated by $k_{1} l_{1}, k_{2} l_{2}\left(k_{1} \neq l_{1}, k_{2} \neq l_{2}\right)$ with $\omega_{k_{1} l_{1}}=\omega_{k_{2} l_{2}}=\omega$. In Sect. 2.1 we gave examples with $\omega^{2}=66$ and 86. Substituting the corresponding 4-mode truncation into Eq. (1) and taking inner products with the eigenfunctions we obtain 4 coupled equations of motion. We divide the equations by $\omega^{2}$, replacing $\omega t$ by $t$ and abbreviate $u_{k_{1} l_{1}}=u_{1}, u_{l_{1} k_{1}}=u_{2}, u_{k_{2} l_{2}}=u_{3}, u_{l_{2} k_{2}}=u_{4}$; we find the system:

$$
\left\{\begin{array}{l}
\ddot{u}_{1}+u_{1}=\varepsilon \frac{3}{4 \omega^{2}} u_{1}\left(\frac{3}{4} u_{1}^{2}+u_{2}^{2}+u_{3}^{2}+u_{4}^{2}\right), \\
\ddot{u}_{2}+u_{2}=\varepsilon \frac{3}{4 \omega^{2}} u_{2}\left(\frac{3}{4} u_{2}^{2}+u_{1}^{2}+u_{3}^{2}+u_{4}^{2}\right), \\
\ddot{u}_{3}+u_{3}=\varepsilon \frac{3}{4 \omega^{2}} u_{3}\left(\frac{3}{4} u_{3}^{2}+u_{1}^{2}+u_{2}^{2}+u_{4}^{2}\right), \\
\ddot{u}_{4}+u_{4}=\varepsilon \frac{3}{4 \omega^{2}} u_{4}\left(\frac{3}{4} u_{4}^{2}+u_{1}^{2}+u_{2}^{2}+u_{3}^{2}\right) .
\end{array}\right.
$$


Note that $3 /\left(4 \omega^{2}\right)$ is a small number, $\varepsilon$ need not be very small. The four normal modes are solutions of system (29); we will show that they are unstable. The system (32) is very symmetric and we can find other exact solutions and invariant manifolds, for instance the 8 periodic solutions given by:

$$
u_{1}^{2}=u_{2}^{2}=u_{3}^{2}=u_{4}^{2} .
$$

The solutions are elliptic periodic functions. A number of invariant manifolds exist:

- 6 ( 2 dof) 4-dimensional invariant manifolds $M_{12}, M_{13}, M_{14}, M_{23}, M_{24}, M_{34}$ with for instance $M_{12}$ given by $u_{3}(t)=u_{4}(t)=0, t \geq 0$. The phase-flow in these invariant manifolds has been described in Sect. 2.2, the normal modes are unstable. Two periodic solutions in general position in $M_{12}$ are given by:

$$
u_{1}(t)=u_{2}(t), \quad \ddot{u}_{1}+u_{1}=\varepsilon \frac{21}{16 \omega^{2}} u_{1}^{3} .
$$

- 4 (3 dof) 6-dimensional invariant manifolds $M_{123}, M_{124}, M_{234}, M_{134}$. Apart from the periodic solutions in 4-dimensional submanifolds we have two periodic solutions in general position given by

$$
\ddot{u}+u=\varepsilon \frac{33}{16 \omega^{2}} u^{3},
$$

with for instance in $M_{123}, u=u_{1}=u_{2}=u_{3}$.

Averaging-normalization of system (32) in polar coordinates (excluding the normal mode planes) we find with $u_{i}(t)=r_{i} \cos \left(t+\psi_{i}\right), \dot{u}_{i}=-r_{i} \sin \left(t+\psi_{i}\right), i=1, \ldots, 4$ :

$$
\left\{\begin{array}{l}
\dot{r}_{1}=-\varepsilon \frac{3}{32 \omega^{2}} r_{1}\left[r_{2}^{2} \sin 2\left(\psi_{1}-\psi_{2}\right)+r_{3}^{2} \sin 2\left(\psi_{1}-\psi_{3}\right)+r_{4}^{2} \sin 2\left(\psi_{1}-\psi_{4}\right)\right], \\
\dot{r}_{2}=-\varepsilon \frac{3}{32 \omega^{2}} r_{2}\left[-r_{1}^{2} \sin 2\left(\psi_{1}-\psi_{2}\right)+r_{3}^{2} \sin 2\left(\psi_{2}-\psi_{3}\right)+r_{4}^{2} \sin 2\left(\psi_{2}-\psi_{4}\right)\right], \\
\dot{r}_{3}=-\varepsilon \frac{3}{32 \omega^{2}} r_{3}\left[-r_{1}^{2} \sin 2\left(\psi_{1}-\psi_{3}\right)-r_{2}^{2} \sin 2\left(\psi_{2}-\psi_{3}\right)+r_{4}^{2} \sin 2\left(\psi_{3}-\psi_{4}\right)\right], \\
\dot{r}_{4}=-\varepsilon \frac{3}{32 \omega^{2}} r_{4}\left[-r_{1}^{2} \sin 2\left(\psi_{1}-\psi_{4}\right)-r_{2}^{2} \sin 2\left(\psi_{2}-\psi_{4}\right)-r_{3}^{2} \sin 2\left(\psi_{3}-\psi_{4}\right)\right] .
\end{array}\right.
$$

The equations for the angles contain the combination angles $\left(\psi_{1}-\psi_{2}\right),\left(\psi_{1}-\psi_{3}\right)$ etc. but we do not need them as in this case we have explicit expressions for the periodic solutions. An integral of the normal form system (35) is:

$$
r_{1}^{2}+r_{2}^{2}+r_{3}^{2}+r_{4}^{2}=2 E_{1},
$$

with constant $E_{1} \geq 0$.

Consider an orbit starting near an unstable normal mode. We expect transitions through several resonance zones. Assume a fixed, positive value $E_{1}$ in Eq. (36). For instance starting near the normal mode plane of the 1st and 3rd mode we have that $r_{1}(0)^{2}+r_{3}(0)^{2}$ will be close to $2 E_{1}$, the other initial conditions are small. The instability will move the orbit on the 7-sphere described by the integral (36) away from the $u_{1}, u_{3}$ normal mode plane. The first resonance zone to encounter will be given by $r_{i}^{2}=E_{1} / 2, i=1, \ldots, 4$, the second one are possibly 4 resonance zones with $r_{i}^{2}=2 E_{1} / 3$, then follow 2 dof resonance zones with $r_{i}^{2}=E_{1}$ after which the recurrence can start. See for illustration Figs. 3 and 4.

\subsection{Embedded Double Resonance in a Detuned Case}

As a rather different case we consider a three mode system generated by $k l=33-57-75$ with $\omega_{33}^{2}=19, \omega_{57}^{2}=\omega_{75}^{2}=75$. So we have a $1: 1$ resonance with a detuned 1:2 reso- 

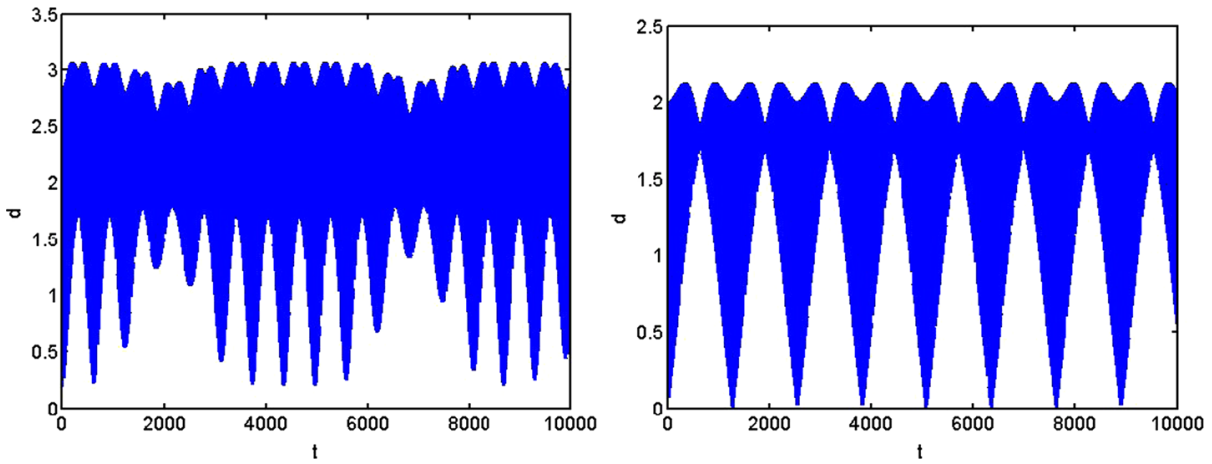

Fig. 3 Recurrence in the two-dimensional Klein-Gordon equation in the case of the 1:1:1:1 resonance based on system (32). (Left) the Euclidean distance $d$ starting near the $u_{1}, u_{3}$ normal mode planes with initial values $u_{1}(0)=1, u_{2}(0)=0.1, u_{3}(0)=-1, u_{4}(0)=0.1$, initial velocities zero; $3 \varepsilon /\left(4 \omega^{2}\right)=0.01$; extending the calculation to 40000 timesteps does not improve the recurrence. The dark areas (on a screen blue) represent large oscillations of the modes resulting in large oscillations of $d$. The details of the case of passage through the resonance zones are shown in Fig. 4. (Right) the case when starting in resonance with initial values $u_{1}(0)=0.5, u_{2}(0)=-0.5, u_{3}(0)=-0.5, u_{4}(0)=0.5$, initial velocities zero; the recurrence is regular
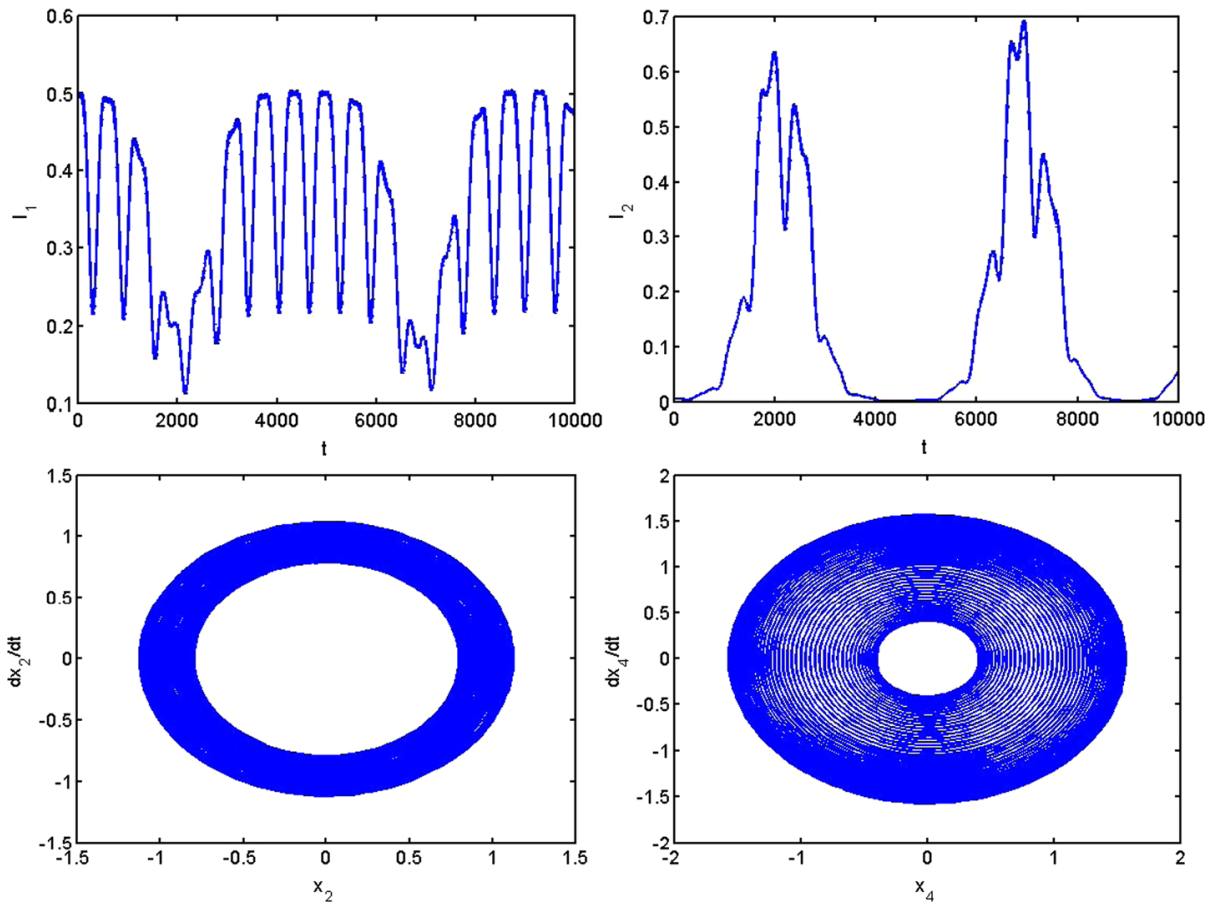

Fig. 4 Details of the flow in the two-dimensional Klein-Gordon equation in the case of the 1:1:1:1 resonance described by system (32) in Fig. 3 left. At the top the actions $I_{1}(t), I_{2}(t)$ showing several passages through resonance. Below projections from 8 -space on the coordinate planes of $x_{2}, \dot{x}_{2}$ and $x_{4}, \dot{x}_{4}$ during a passage through resonance for $2000 \leq t \leq 2500$. The orbits of modes 2 and 4 are winding around tori in the resonance zone 
nance. We propose the three-terms Galerkin truncation with again Dirichlet boundary conditions:

$$
u_{33}(t) \sin 3 x \sin 3 y+u_{57}(t) \sin 5 x \sin 7 y+u_{75}(t) \sin 7 x \sin 5 y .
$$

Substituting this truncation into Eq. (1), taking inner products with the eigenfunctions and replacing $\sqrt{75} t$ by $t$ we find the three dof system:

$$
\left\{\begin{array}{l}
\ddot{u}_{33}+\frac{1}{4} u_{33}=-\frac{1}{300} u_{33}+\varepsilon \frac{1}{100}\left(\frac{3}{4} u_{33}^{3}+u_{33} u_{57}^{2}+u_{33} u_{75}^{2}\right), \\
\ddot{u}_{57}+u_{57}=\varepsilon \frac{1}{100}\left(\frac{3}{4} u_{57}^{3}+u_{33}^{2} u_{57}+u_{57} u_{75}^{2}\right), \\
\ddot{u}_{75}+u_{75}=\varepsilon \frac{1}{100}\left(\frac{3}{4} u_{75}^{3}+u_{33}^{2} u_{75}+u_{57}^{2} u_{75}\right) .
\end{array}\right.
$$

The three normal modes are solutions of system (38). Putting $u_{33}(t)=\dot{u}_{33}(t)=0$, the system has a two dof (4-dimensional) invariant manifold with 1:1 dynamics as before. In a similar way we find the invariant manifold $M_{12}$ if the third mode vanishes and $M_{13}$ if the second mode vanishes. The frequency ratios are $\frac{1}{2}: 1: 1$ so mode 1 is close to $1: 2$ resonance with the other two modes. However, as we shall see, the 1:1 resonance of the modes 2 and 3 dominates the flow outside the resonance zones and the normal mode planes. The size of $\varepsilon$ and the chosen energy level determine the detuning effect. Excluding the normal modes, first order averaging-normalization for amplitudes and phases $r, \psi$ from (7) produces with $\chi_{1}=\psi_{2}-\psi_{3}$ :

$$
\begin{cases}\dot{r}_{1}=0, & \dot{\psi}_{1}=\frac{1}{300}-\varepsilon \frac{1}{200}\left(\frac{9}{8} r_{1}^{2}+r_{2}^{2}+r_{3}^{2}\right), \\ \dot{r}_{2}=-\varepsilon \frac{1}{800} r_{2} r_{3}^{2} \sin 2 \chi_{1}, & \dot{\psi}_{2}=-\varepsilon \frac{1}{400}\left(\frac{9}{8} r_{2}^{2}+r_{1}^{2}+r_{3}^{2}\left(1+\frac{1}{2} \cos 2 \chi_{1}\right)\right), \\ \dot{r}_{3}=+\varepsilon \frac{1}{800} r_{2}^{2} r_{3} \sin 2 \chi_{1}, & \dot{\psi}_{3}=-\varepsilon \frac{1}{400}\left(\frac{9}{8} r_{3}^{2}+r_{1}^{2}+r_{2}^{2}\left(1+\frac{1}{2} \cos 2 \chi_{1}\right)\right) .\end{cases}
$$

We find again the integral

$$
r_{2}^{2}+r_{3}^{2}=2 E_{1}, \quad E_{1} \geq 0
$$

and similar to the case of the first three modes:

$$
\frac{d \chi_{1}}{d t}=-\varepsilon \frac{1}{800}\left(r_{2}^{2}-r_{3}^{2}\right)\left(\frac{1}{4}-\cos 2 \chi_{1}\right) .
$$

Putting $\cos \chi_{1}=1 / 4$ we find stable and unstable invariant manifolds, in fact heteroclinics, of the $u_{57}, u_{75}$ normal modes given by

$$
\dot{r}_{2}=-\frac{\varepsilon}{800} r_{2}^{3} \sin 2 \chi_{1}, \quad \dot{r}_{3}=\frac{\varepsilon}{800} r_{3}^{3} \sin 2 \chi_{1}, \quad \sin 2 \chi_{1}= \pm \frac{\sqrt{15}}{4} .
$$

In the primary resonance zones $M_{1}, M_{2}$ where by $r_{2}=r_{3}, \sin 2 \chi_{1}=0$ we have for the periodic solutions:

$$
r_{2}=r_{3}=E_{1}, \quad \chi_{1}=0, \frac{\pi}{2}, \pi, \frac{3 \pi}{2} .
$$

In Fig. 5 we have chosen the initial conditions near the unstable $u_{75}$ normal mode. This causes repeated passage through the primary resonance zones. In Fig. 5 we have left the case of nearly pure $1: 1$ resonance (choosing $u_{33}=0$ would eliminate the mode $u_{33}$ completely). The middle and right figure shows the influence of secondary resonances (embedded double resonance) that complicate passage through the zones.

In the averaging-normal form (39) the 1:2 resonance is not active. As we will show, at higher order we find in the invariant manifolds $M_{12}, M_{13}$ the 2:4 resonance with combination 

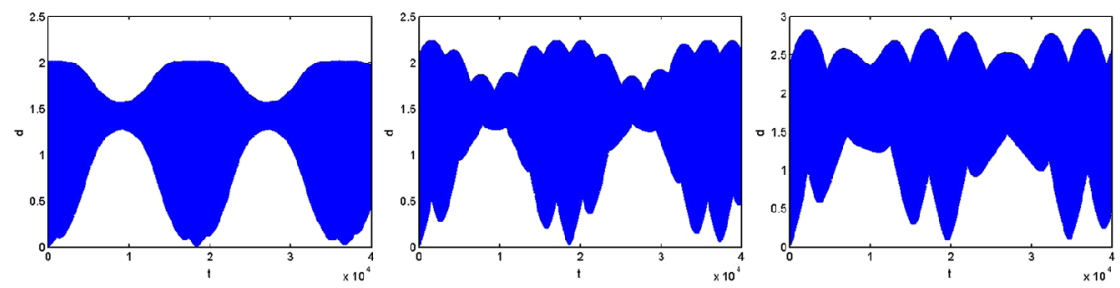

Fig. 5 Recurrence in the two-dimensional Klein-Gordon equation for a detuned case with 3 modes. The Euclidean distance $d$ to the initial values $u_{33}(0), u_{57}(0)=0.1, u_{75}(0)=1$, initial velocities zero and $\varepsilon=0.5$, for system (38) is shown in the case of passage through the resonance zones. (Left) the case $u_{33}(0)=0.1$, middle figure $u_{33}(0)=0.5$; recurrence for $d \leq 0.1$ takes 18000 timesteps in both cases. (Right) $u_{33}(0)=1$ showing different recurrence behavior. The action $I_{1}$ shows very small variations (around 0.001 ) in all cases
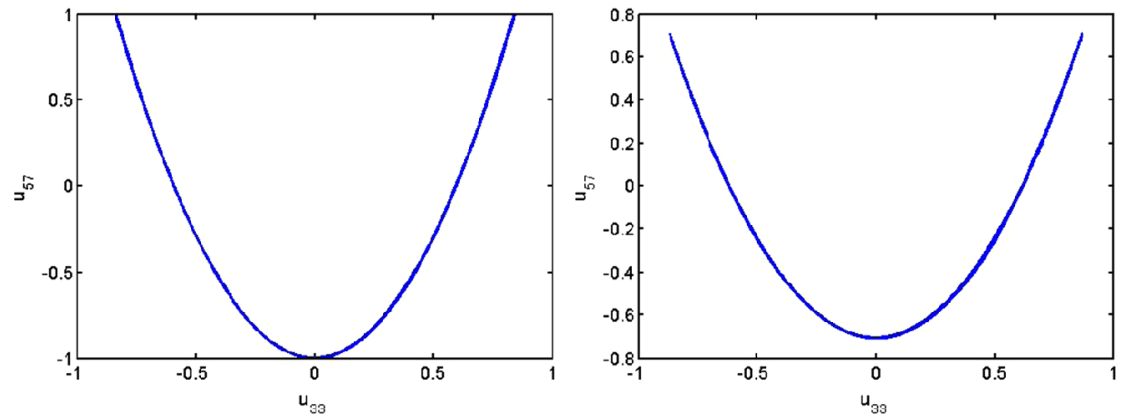

Fig. 6 Projections of the periodic solutions in the secondary resonance zones of the $u_{33}, u_{57}, u_{75}$ system (38) with $\varepsilon=0.5$. In the resonance zones we project $u_{33}(t), u_{57}(t)$ showing a quadratic curve that is typical for the higher order resonance. (Left) the two dof submanifold $M_{12}: u_{75}(0)=\dot{u}_{75}(0)=0$ with $u_{33}(0)=0.838, u_{57}(0)=1$ (so $E_{1}=0.5$ ); the resonance is $2: 4$. (Right) the case $u_{33}(0)=0.87$, $u_{57}(0)=0.707, u_{75}(0)=0.707$, initial velocities zero (so $\chi_{1}=0, E_{1}=0.5$ ). The choice $\chi_{1}=\pi$ produces the same picture

angles:

$$
\chi_{2}=4 \psi_{1}-2 \psi_{2}, \quad \chi_{3}=4 \psi_{1}-2 \psi_{3} .
$$

In the resonance zones outside the normal mode planes the 2:4:4 resonance will arise.

Consider for instance $M_{12}$; we find from the first order normal form system (39):

$$
\dot{r}_{1}=0, \quad \dot{\psi}_{1}=\frac{1}{300}-\varepsilon \frac{1}{200}\left(\frac{9}{8} r_{1}^{2}+r_{2}^{2}\right), \quad \dot{r}_{2}=0, \quad \dot{\psi}_{2}=-\varepsilon \frac{1}{400}\left(\frac{9}{8} r_{2}^{2}+r_{1}^{2}\right) .
$$

We have no exchange of energy at this first order approximation. Consider the combination angle:

$$
\frac{d \chi_{2}}{d t}=4 \dot{\psi}_{1}-2 \dot{\psi}_{2}=\frac{1}{25}\left(\frac{1}{3}-\frac{\varepsilon}{64}\left(27 r_{1}^{2}+23 r_{2}^{2}\right)\right) .
$$

If $\chi_{2}$ is timelike, we can remove more terms by averaging, secondary resonance will not be relevant. This is the case if $d \chi_{2} / d t$ has no zeros. Values of $\varepsilon, r_{1}, r_{2}$ for which the righthand side of Eq. (43) vanishes can produce a resonance manifold in $M_{12}$ with higher order periodic solutions; see Fig. 6. To characterize these higher order solutions in $M_{12}, M_{13}$ and in 
the 3-dof resonance zones we compute the normal form to next order. To show the role of detuning we replace $1 / 300$ by $\delta$. We find to second order:

$$
\left\{\begin{aligned}
\dot{r}_{1}= & -\varepsilon^{2} a_{1} r_{1}^{3}\left(r_{2}^{2} \sin \chi_{2}+r_{3}^{2} \sin \chi_{3}\right), \\
\dot{\psi}_{1}= & \delta-\varepsilon \frac{1}{200}\left(\frac{9}{8} r_{1}^{2}+r_{2}^{2}+r_{3}^{2}\right)-\delta^{2}+\varepsilon \delta\left(a_{2} r_{1}^{2}+a_{3} r_{2}^{2}+a_{3} r_{3}^{2}\right)-\varepsilon^{2}\left(a_{4} r_{1}^{4}+a_{5} r_{2}^{4}+a_{5} r_{3}^{4}\right. \\
& \left.+a_{6} r_{1}^{2} r_{2}^{2}+a_{6} r_{1}^{2} r_{3}^{2}+a_{7} r_{2}^{2} r_{3}^{2}+a_{8} r_{1}^{2} r_{2}^{2} \cos \chi_{2}+a_{8} r_{1}^{2} r_{3}^{2} \cos \chi_{3}+a_{9} r_{2}^{2} r_{3}^{2} \cos 2 \chi_{1}\right), \\
\dot{r}_{2}=- & \varepsilon \frac{1}{800} r_{2} r_{3}^{2} \sin 2 \chi_{1}+\varepsilon^{2}\left(\frac{1}{4} a_{1} r_{1}^{4} r_{2} \sin \chi_{2}-\left(a_{10} r_{1}^{2} r_{2} r_{3}^{2}+a_{11} r_{2}^{3} r_{3}^{2}+a_{11} r_{2} r_{3}^{4}\right) \sin 2 \chi_{1}\right), \\
\dot{\psi}_{2}= & -\varepsilon \frac{1}{400}\left(\frac{9}{8} r_{2}^{2}+r_{1}^{2}+r_{3}^{2}\left(1+\frac{1}{2} \cos 2 \chi_{1}\right)\right)+a_{12} \varepsilon \delta r_{1}^{2}-\varepsilon^{2}\left(a_{13} r_{1}^{4}+a_{14} r_{1}^{2} r_{2}^{2}+a_{15} r_{2}^{4}\right. \\
& \left.+a_{16} r_{1}^{2} r_{3}^{2}+a_{17} r_{2}^{2} r_{3}^{2}+a_{18} r_{3}^{4}+a_{19} r_{1}^{4} \cos \chi_{2}+r_{3}^{2}\left(a_{20} r_{1}^{2}+2 a_{21} r_{2}^{2}+a_{21} r_{3}^{2}\right) \cos 2 \chi_{1}\right), \\
\dot{r}_{3}= & +\varepsilon \frac{1}{800} r_{2}^{2} r_{3} \sin 2 \chi_{1}+\varepsilon^{2}\left(\frac{1}{4} a_{1} r_{1}^{4} r_{3} \sin \chi_{3}+\left(a_{10} r_{1}^{2} r_{2}^{2} r_{3}+a_{11} r_{2}^{4} r_{3}+a_{11} r_{2}^{2} r_{3}^{3}\right) \sin 2 \chi_{1}\right), \\
\dot{\psi}_{3}= & -\varepsilon \frac{1}{400}\left(\frac{9}{8} r_{3}^{2}+r_{1}^{2}+r_{2}^{2}\left(1+\frac{1}{2} \cos 2 \chi_{1}\right)\right)+a_{12} \varepsilon \delta r_{1}^{2}-\varepsilon^{2}\left(a_{13} r_{1}^{4}+a_{16} r_{1}^{2} r_{2}^{2}+a_{18} r_{2}^{4}\right. \\
& \left.+a_{14} r_{1}^{2} r_{3}^{2}+a_{17} r_{2}^{2} r_{3}^{2}+a_{15} r_{3}^{4}+a_{19} r_{1}^{4} \cos \chi_{3}+r_{2}^{2}\left(a_{20} r_{1}^{2}+a_{21} r_{2}^{2}+2 a_{21} r_{3}^{2}\right) \cos 2 \chi_{1}\right) .
\end{aligned}\right.
$$

The constants $a_{1}, \ldots, a_{21}$ are positive. System (44) has the integral

$$
\frac{1}{4} r_{1}^{2}+r_{2}^{2}+r_{3}^{2}=\text { constant }
$$

It is clear from system (44) that the 1:1 resonance of the 2 nd and 3rd modes dominates the flow outside the resonance zones $M_{1}, M_{2}$ and outside the invariant manifolds $M_{12}, M_{13}$.

Normalization in $\boldsymbol{M}_{\mathbf{1 2}}$ With vanishing 3rd mode in system (38) the 2nd order normal form in $M_{12}$ becomes:

$$
\left\{\begin{aligned}
\dot{r}_{1}= & -\varepsilon^{2} a_{1} r_{1}^{3} r_{2}^{2} \sin \chi_{2}, \\
\dot{\psi}_{1}= & \delta-\varepsilon \frac{1}{200}\left(\frac{9}{8} r_{1}^{2}+r_{2}^{2}\right)-\delta^{2}+\varepsilon \delta\left(a_{2} r_{1}^{2}+a_{3} r_{2}^{2}\right) \\
& \quad-\varepsilon^{2}\left(a_{4} r_{1}^{4}+a_{5} r_{2}^{4}+a_{6} r_{1}^{2} r_{2}^{2}+a_{8} r_{1}^{2} r_{2}^{2} \cos \chi_{2}\right), \\
\dot{r}_{2}= & \varepsilon^{2} \frac{1}{4} a_{1} r_{1}^{4} r_{2} \sin \chi_{2}, \\
\dot{\psi}_{2}= & -\varepsilon \frac{1}{400}\left(\frac{9}{8} r_{2}^{2}+r_{1}^{2}\right)+a_{12} \varepsilon \delta r_{1}^{2}-\varepsilon^{2}\left(a_{13} r_{1}^{4}+a_{14} r_{1}^{2} r_{2}^{2}+a_{15} r_{2}^{4}+a_{19} r_{1}^{4}\right) \cos \chi_{2} .
\end{aligned}\right.
$$

For $M_{13}$ we have analogous results. If $\sin \chi_{2}=0$ and remains zero in time, the amplitudes $r_{1}, r_{2}$ are constant in time. We have in $M_{12}$ :

$$
\dot{\chi}_{2}=4 \delta-\frac{\varepsilon}{400}\left(7 r_{1}^{2}+\frac{23}{4} r_{2}^{2}\right)+\cdots
$$

where the dots contain terms of $O\left(\delta^{2}, \varepsilon \delta, \varepsilon^{2}\right)$ with arguments $r_{1}, r_{2}, \cos \chi_{2}$. A necessary condition for the presence of such solutions is

$$
4 \delta-\frac{\varepsilon}{400}\left(7 r_{1}^{2}+\frac{23}{4} r_{2}^{2}\right)=0 .
$$


if $\delta \leq 0$ there are no such solutions. Assume $\delta>0$; this produces from the necessary condition a relation between $\delta, \varepsilon, r_{2}$ and $r_{2}$. The second condition is that $\dot{\chi}_{2}=0$ at next order. This produces with $\cos \chi_{2}= \pm 1$ another equation between the same quantities. If, together with the energy integral, we can solve these 3 equations, we have determined a resonance manifold in $M_{12}$ where a 4:2 resonant periodic solution between the first two modes in $M_{12}$ can be found. For a choice of parameters such a solution is shown in Fig. 6 (left).

Normalization in a General Position Resonance Zone In the primary resonance zones we have obtained to first order $r_{2}^{2}=r_{3}^{2}=E_{1}, \sin 2 \chi_{1}=0$. For the amplitudes we find to second order from system (44) with

$$
\begin{aligned}
& \sin \chi_{2}+\sin \chi_{3}=2 \sin \chi_{4} \cos 2 \chi_{1}, \quad \chi_{4}=\left(4 \psi_{1}-\psi_{2}-\psi_{3}\right), \quad \cos 2 \chi_{1}= \pm 1: \\
& \left\{\begin{array}{l}
\dot{r}_{1}=-\varepsilon^{2} a_{1} r_{1}^{3} E_{1} \sin \chi_{4}+\cdots, \\
\dot{r}_{2}=\varepsilon^{2} \frac{a_{1}}{4} r_{1}^{4} \sqrt{E_{1}} \sin \chi_{2}+\cdots, \\
\dot{r}_{2}=\varepsilon^{2} \frac{a_{1}}{4} r_{1}^{4} \sqrt{E_{1}} \sin \chi_{3}+\cdots
\end{array}\right.
\end{aligned}
$$

with the dots representing higher order terms. We consider the combination angle $\chi_{4}$ characterizing a possible resonance manifold in general position (all modes non-zero). In a domain in a primary resonance zone where $\sin \chi_{4}=0$ and $\dot{\chi}_{4}=0$ we expect to find a 1:2:2 periodic solution. Note that it suffices to require $\sin \chi_{2}=\sin \chi_{3}=0$. We find from system (44) to first order in 2 resonance zones:

$$
\begin{cases}\frac{d \chi_{4}}{d t}=\frac{1}{75}-\varepsilon \frac{1}{400}\left(7 r_{1}^{2}+\frac{43}{4} E_{1}\right) & \text { if } \cos 2 \chi_{1}=1, \\ \frac{d \chi_{4}}{d t}=\frac{1}{75}-\varepsilon \frac{1}{400}\left(7 r_{1}^{2}+\frac{51}{4} E_{1}\right) & \text { if } \cos 2 \chi_{1}=-1\end{cases}
$$

In the case of Fig. 5 and putting $\varepsilon=0.5$ in (39) as the coefficients are very small we have from (49) zeros of the righthand side producing secondary resonances in the primary resonance zones. We find $\cos 2 \chi_{1}=1, r_{1}(0)=0.87$ and $\cos 2 \chi_{1}=-1, r_{1}(0)=0.78$. To show more evidence for the existence of secondary resonance and the presence of tori that influence the recurrence as in fig, 5, consider Fig. 6. The theory of higher order resonance predicts two stable 1:2:2 periodic solutions and two unstable ones. In Fig. 6 we depict one of the cases.

Exact solutions as in Sect. 2.2.

Apart from the normal modes we find from system (38) the exact solutions $u_{57}^{2}(t)=$ $u_{75}^{2}(t)$. The corresponding equation for $u_{57}$ is:

$$
\ddot{u}_{57}+u_{57}=\varepsilon \frac{1}{100} u_{57}\left(\frac{7}{4} u_{57}^{2}+u_{33}^{2}\right) .
$$

With $J(t)$ the angular momentum (21) interaction for $u_{57}$ and $u_{75}$ we have

$$
\frac{d J}{d t}=-\varepsilon \frac{1}{400} u_{57} u_{75}\left(u_{57}^{2}-u_{75}^{2}\right) .
$$

Again, the critical points of the angular momentum equation correspond with the resonance zones. 


\section{Appendix on the Analysis of Detuning}

Our results on normalization and error estimates are based on [12] with slight modifications. We assume existence and uniqueness of solutions of initial value problems, sufficient smoothness and $T$-periodicity of vector fields. To study the dynamics of secondary resonance in the detuned case, we have to normalize to higher order, in Hamiltonian terms to $H_{6}$. Detuning produces in a number of cases interesting bifurcation phenomena, so we outline the procedure here in a rather general context.

The usual practice is to put the equations of motion in the standard form for averagingnormalization using transformation (8):

$$
\dot{x}=\varepsilon f(t, x)+\varepsilon^{2} g(t, x)+\varepsilon^{3} \ldots, \quad x(0)=x_{0} .
$$

This transformation assumes that we stay away from the normal modes where the amplitudes vanish. In our paper this poses no problem as in the Galerkin projections of the cubic KleinGordon equation the normal modes are exact solutions. Assume that the vector fields $f, g$ are $T$-periodic and consider the averaged vector field

$$
f^{0}(x)=\frac{1}{T} \int_{0}^{T} f(s, x) d s
$$

and the initial value problem

$$
\dot{y}=\varepsilon f^{0}(y), \quad y(0)=x_{0},
$$

then $x(t)-y(t)=O(\varepsilon)$ on the timescale $1 / \varepsilon$. Suppose we need a higher order approximation. We will use the near-identity transformation:

$$
x=z+\varepsilon u^{1}(t, z)
$$

with

$$
u^{1}(t, z)=\int_{0}^{t}\left(f(s, z)-f^{0}(z)\right) d s .
$$

Introduce the vector field

$$
f_{1}(t, z)=\nabla f(t, z) u^{1}(t, z)
$$

with $f_{1}^{0}$ the average of $f_{1}$, then the next approximation of Eq. (50) is obtained as follows. Solve the initial value problem

$$
\dot{w}=\varepsilon f^{0}(w)+\varepsilon^{2}\left(f_{1}^{0}(w)+g^{0}(w)\right), \quad w(0)=x_{0},
$$

then $x(t)=w(t)+\varepsilon u^{1}(t, w(t))+O\left(\varepsilon^{2}\right)$ on the timescale $1 / \varepsilon$.

Detuning introduces a second or even more small parameters. We explain this in the context of this paper, the idea is quite general. Consider the $2 n$-dimensional equations of motion for $x=\left(x_{1}, x_{2}, \ldots, x_{n}\right)$ :

$$
\ddot{x}_{i}+\omega_{i}^{2} x_{i}=\varepsilon F(x, \dot{x}), \quad i=1, \ldots, n .
$$

The frequencies $\omega_{i}$ are close to the resonant frequencies $\Omega_{i}, i=1, \ldots, n$. We rewrite system (53) as:

$$
\ddot{x}_{i}+\Omega_{i}^{2} x_{i}=\delta_{i} x_{i}+\varepsilon F(x, \dot{x}), \quad i=1, \ldots, n .
$$


The $n$ parameters $\delta_{i}$ are supposed to be small; they do not depend on $\varepsilon$ but still, the parameters $\varepsilon, \delta_{i}$ have to be compared in size. If $\varepsilon \ll \delta_{i}$, the first order approximation does not involve $F(x)$. Assuming that $\delta_{i}, i=1, \ldots, n$ is of size $\varepsilon$ both parameters play a part at first and second order. Applying transformation (8) to system (54) we find for $i=1, \ldots, n$ :

$$
\left\{\begin{aligned}
\dot{r}_{i}=- & \frac{1}{\Omega_{i}} \sin \left(\Omega_{i} t+\psi_{i}\right) \\
& \times\left(\delta_{i} r_{i} \cos \left(\Omega_{i} t+\psi_{i}\right)+\varepsilon F\left(r_{i} \cos \left(\Omega_{i} t+\psi_{i}\right),-\Omega_{i} r_{i} \sin \left(\Omega_{i} t+\psi_{i}\right)\right)\right), \\
\dot{\psi}_{i}= & -\frac{1}{\Omega_{i} r_{i}} \cos \left(\Omega_{i} t+\psi_{i}\right) \\
& \times\left(\delta_{i} r_{i} \cos \left(\Omega_{i} t+\psi_{i}\right)+\varepsilon F\left(r_{i} \cos \left(\Omega_{i} t+\psi_{i}\right),-\Omega_{i} r_{i} \sin \left(\Omega_{i} t+\psi_{i}\right)\right)\right) .
\end{aligned}\right.
$$

Averaging over the common period $T$ we find for $i=1, \ldots, n, r=r_{1}, \ldots, r_{n}, \psi=$ $\psi_{1}, \ldots, \psi_{n}$ :

$$
\dot{r}_{i}^{0}=\varepsilon f_{i}^{0}(r, \psi), \quad \dot{\psi}_{i}^{0}=-\frac{\delta_{i}}{2 \Omega_{i}}+\varepsilon f_{i}^{0}(r, \psi)
$$

We can apply near-identity transformation (51) to system (55) to obtain a second order approximation; the resulting system to solve will be of the form (52) with terms added of size $\delta_{i}$ and $\delta_{i}^{2}$.

Applying higher order normalization to a detuned resonance like system (38) we find at $O\left(\varepsilon^{2}\right)$ terms of the form $r_{1}^{3} r_{2}^{2} \sin \left(4 \psi_{1}-2 \psi_{2}\right)$ and similar terms involving $r_{3}$. These terms introduce the $4: 2$ resonances with corresponding periodic solutions discussed in the preceding section.

\section{Conclusions}

1. In mathematical physics PDEs have often been analyzed in the case of one space dimension. We have shown that when allowing more space dimensions, the results may change remarkably. We discuss a typical case of mathematical physics, the cubic Klein-Gordon equation.

2. The validity of asymptotic approximations holds on intervals of time proportional to $1 / \varepsilon$ and $\omega_{k l}$. Our analysis yields some conclusions using the truncation procedure of series (3). First, if $\omega_{k l}$ is large enough the tail of the series will take an extremely long time to become effective. Secondly, the modes in 1:1 resonance are ubiquitous but combination of independent 1:1 systems produces no new phenomena. Thirdly, interesting phenomena like embedded double resonance arise from detuning with new resonant interactions. There will exist an infinite number of such detuned systems, but they arise for large values of $\omega_{k l}$.

For the complicated dynamics described in the preceding sections to be observed one has to choose the corresponding initial conditions producing the resonant modes. Exciting for instance only one mode, there will be nontrivial evolution if this mode is unstable in a resonant setting and if it is slightly perturbed.

3. The most interesting dynamics is described for the 1:1:1:1 resonance in Sect. 2.6 and the detuned resonance in Sect. 2.7. The resonance zones vanish (have size $o(1)$ ) as $\varepsilon \rightarrow 0$ and so are free boundary layers in the sense of singular perturbation theory. In these zones the resonances produce locally stable and unstable periodic solutions with corresponding stable and unstable manifolds. Intersection of invariant manifolds associated with the periodic solutions in the resonance zones are in Hamiltonian mechanics the main source 
of chaos. For small values of $\varepsilon$ this will enable the possibility of boundary layer chaos in the cubic Klein-Gordon equation, it may be more prominent if $\varepsilon$ increases.

4. Changing the boundary conditions or the shape of the domain will of course change our results. However, the set-up of our analysis is typical for such new problems. Symmetries, for instance considering circle or ring domains, may simplify the analysis. New phenomena may arrive when studying non-convex domains.

Acknowledgement A calculation by hand gave the presence of $\chi_{2}$ and $\chi_{3}$ terms in the second order normal form of Sect. 2.7. In a next step second order normalization producing system (44) was carried out by Taoufik Bakri using MATHEMATICA.

Publisher's Note Springer Nature remains neutral with regard to jurisdictional claims in published maps and institutional affiliations.

Open Access This article is distributed under the terms of the Creative Commons Attribution 4.0 International License (http://creativecommons.org/licenses/by/4.0/), which permits unrestricted use, distribution, and reproduction in any medium, provided you give appropriate credit to the original author(s) and the source, provide a link to the Creative Commons license, and indicate if changes were made.

\section{References}

1. Bambusi, D.: Birkhoff normal form for some nonlinear PDEs. Commun. Math. Phys. 234, 253-285 (2003)

2. Bambusi, D.: Galerkin averaging method and Poincaré normal form for some quasilinear PDEs. Ann. Sc. Norm. Super. Ser. 4 4, 669-702 (2005)

3. Efthymiopoulos, C., Harsoula, M.: The speed of Arnold diffusion. Physica D 251, 19-38 (2013)

4. Fečkan, M.: A Galerkin-averaging method for weakly nonlinear equations. Nonlinear Anal. 41, 345-369 (2000)

5. Guzzo, M., Lega, E.: The Nekhoroshev theorem and the observation of long-term diffusion in Hamiltonian systems. Regul. Chaotic Dyn. 21, 707-719 (2016)

6. Krol, M.S.: On a Galerkin-averaging method for nonlinear, undamped continuous systems. Math. Methods Appl. Sci. 11, 649-664 (1989)

7. Lardner, R.W.: Asymptotic solutions of nonlinear wave equations using the methods of averaging and two-timing. Q. Appl. Math. 35, 225-238 (1977)

8. Nekhoroshev, N.: An exponential estimate of the time of stability of nearly integrable Hamiltonian systems. Russ. Math. Surv. 32, 1-65 (1977)

9. Pals, H.: The Galerkin-averaging method for the Klein-Gordon equation in two space dimensions. Nonlinear Anal. 27, 841-856 (1996)

10. Poincaré, H.: Les Méthodes Nouvelles de la Mécanique Célèste, 3 vols. Gauthier-Villars, Paris (1892, 1893, 1899)

11. Sanders, J.A.: Are higher order resonances really interesting? Celest. Mech. 16, 421-440 (1977)

12. Sanders, J.A., Verhulst, F., Murdock, J.: Averaging Methods in Nonlinear Dynamical Systems. Springer, Berlin (2007)

13. Tuwankotta, J.M., Verhulst, F.: Symmetry and resonance in Hamiltonian systems. SIAM J. Appl. Math. 61, 1369-1385 (2000)

14. Verhulst, F.: Discrete symmetric dynamical systems at the main resonances with applications to axisymmetric galaxies. Philos. Trans. R. Soc. A 290, 435-465 (1979)

15. Verhulst, F.: Near-integrability and recurrence in FPU-cells. Int. J. Bifurc. Chaos 26(14), 1650230 (2016). https://doi.org/10.1142/S0218127416502308

16. Verhulst, F.: Interaction of low and higher order Hamiltonian resonances. Int. J. Bifurc. Chaos 28, 8 (2018). https://doi.org/10.1142/S0218127418500979 